\title{
HEMATURIA MASIVA POR FISTULA ARTERIO-URETERAL
}

\author{
J.A. SUAREZ GONZÁLEZ, M. VEREZ VIVERO, A.V. NÚÑEZ LÓPEZ \\ Servicio de Urología. Hospital da Costa. Burela. Lugo.
}

Actas Urol Esp. 28 (9): 680-682, 2004

\section{RESUMEN}

HEMATURIA MASIVA POR FÍSTULA ARTERIO-URETERAL

Presentamos un caso de hematuria masiva por fístula arterio-ureteral debido a complicación urológica de la cirugía vascular protésica.

Este tipo de fístulas son una causa rara de hematuria macroscópica masiva y la presentación clínica más común es la hematuria intermitente.

El diagnóstico es difícil y debe sospecharse clínicamente, siendo la confirmación mediante pruebas de imagen.

La única posibilidad terapéutica es quirúrgica.

PALABRAS CLAVE: Fístula arterio-ureteral. Hematuria. Cirugía vascular.

\section{ABSTRACT \\ MASSIVE HEMATURIA FROM ARTERY-URETERAL FISTULA}

We present a case of massive hematuria from artery-ureteral fistula due to urologic complication of the protesic vascular surgery.

These kinds of fistula are a rare case of massive macroscopic hematuria and the commonest clinical presentation is the intermittent hematuria.

The only therapeutic possibility is surgical.

KEY WORDS: Artery-ureteral fistula. Hematuria. Vascular surgery.

$\mathrm{L}$

a fistula arterio-ureteral es una causa rara de hematuria macroscópica masiva debiéndose algunos casos a la cirugía vascular previa ${ }^{1}$. Característicamente cursa como hematuria intermitente $\mathrm{y}$ debe sospecharse en pacientes con antecedentes de cirugía vascular o pélvica, instrumentación endoureteral, radioterapia o patología vascular ${ }^{2}$. El diagnóstico debe confirmarse mediante urografia intravenosa (UIV), ureteropielografía retrógrada, arteriografía o tomografía axial computerizada (TAC) ${ }^{3}$. La única posibilidad terapéutica es quirúrgica ${ }^{1}$.

Presentamos un caso de hematuria macroscópica incoercible en paciente al que previamente se la practicó un by-pass aorto-ilíaco derecho por isquemia de miembro inferior derecho. Se describe la actuación diagnóstica y se citan las posibles opciones terapéuticas.

\section{CASO CLÍNICO}

Paciente de 86 años, con buen estado general y sin alergias medicamentosas conocidas, que presenta los siguientes antecedentes personales: exfumador, bebedor moderado, bronquitis crónica, accidente cerebro-vascular agudo con hemiparesia residual desde hace 10 años, insuficiencia cardiorrespiratoria, By-pass aortoilíaco derecho.

Desde el punto de vista urológico, prostatismo de varios años de evolución, siendo portador de 
sonda vesical a permanencia y hematuria macroscópica intermitente de un mes de evolución.

Se trata por tanto de un enfermo con pluripatologia que ingresa en nuestro servicio por un cuadro de hematuria macroscópica con coágulos, no obstructiva pero sí anemizante e incoercible del que únicamente conocemos, a pesar de su edad, que presenta un prostatismo por hipertrofia prostática y una atrofia renal derecha secundaria a dilatación de causa no filiada.

Dada la intensidad de la hematuria fue preciso instaurar lavado vesical continuo de forma permanente así como realizar varias hemotransfusiones (cifras de hemoglobina iniciales de 6 $\mathrm{g} / \mathrm{dl}$ ) no logrando cifras postransfusionales superiores a $\operatorname{los} 9 \mathrm{~g} / \mathrm{dl}$.

Entre los estudios realizados, las citologías miccionales resultaron negativas para carcinoma urotelial. La ecografía urológica únicamente nos informa de la uropatía derecha ya conocida y de la hipertrofia prostática con signos de "vejiga de lucha". Se realiza uretrocistoscopia bajo anestesia, objetivándose marcado crecimiento endouretral de ambos lóbulos prostáticos con lóbulo medio prominente, vejiga trabeculada sin neoformaciones exofiticas ni zonas sospechosas, orificio ureteral derecho de forma y situación normales pero con emisión de abundantes coágulos filiformes.

Ante este hallazgo y con la sospecha de tumor de vias, se intenta realizar ureteropielografía ascendente derecha sin éxito por no progresión del catéter y reflujo del contraste.

El siguiente paso fue la realización de UIV (Fig. 1), que dada su función renal (creatinina entre 2 y $3 \mathrm{mg} / \mathrm{dl}$.) resultó también insuficiente para diagnóstico, mostrando una pobre eliminación de contraste por el riñón izquierdo y anulación funcional derecha.

Dado que con las pruebas anteriores no se aclaró la causa de la hematuria, se realizó TAC abdominopélvico (Figs. 2) en el que se observa uropatía obstructiva derecha con atrofia renal y pseudoaneurisma de arteria iliaca externa derecha, de unos $6 \mathrm{~cm}$ que implica al tercio distal del uréter derecho (sin poder descartar infiltración del mismo) presentando en su interior una imagen correspondiente a una prótesis vascular. Ante este hallazgo, se realiza Eco-doppler color

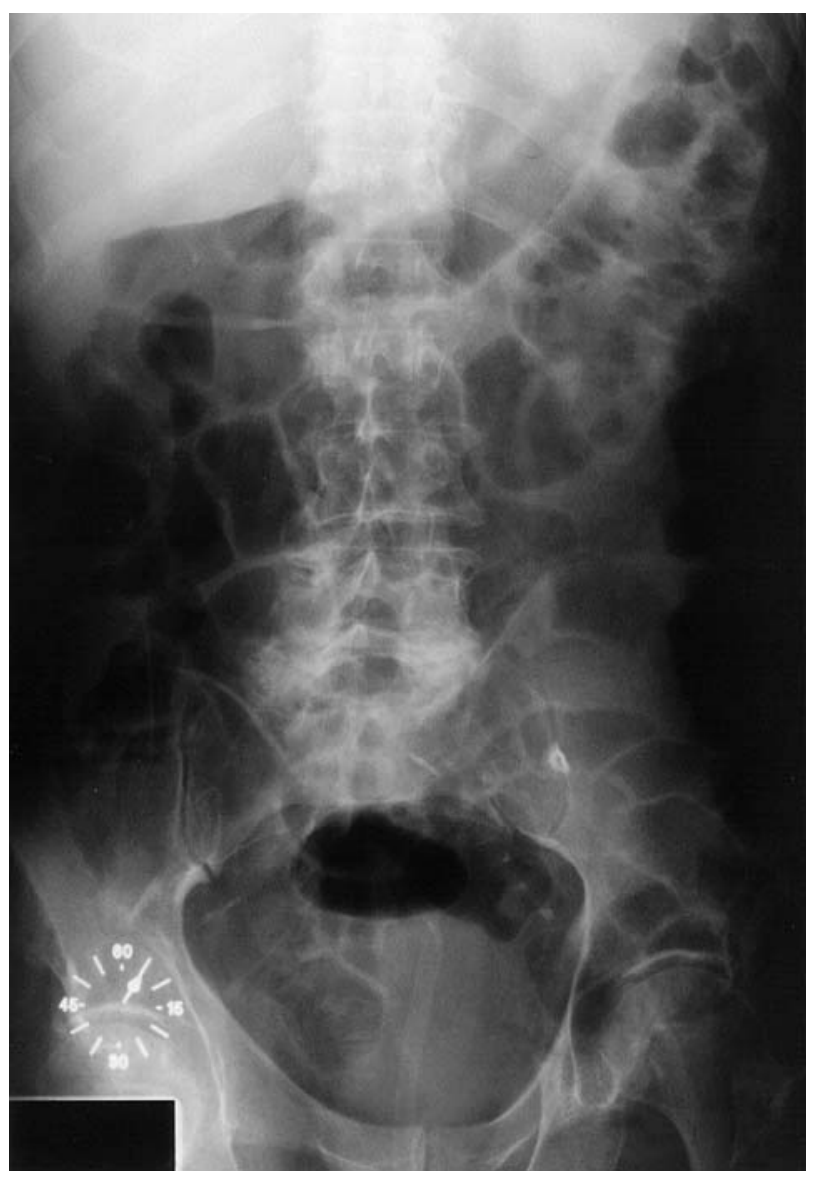

FIGURA 1. Urografia intravenosa donde se observa una pobre eliminación de contraste por el riñón izquierdo y anulación funcional derecha.

donde se observa flujo sanguíneo a través de una fístula arterio-ureteral.

Nuestra opción terapéutica hubiera sido la colocación de una endoprótesis de no haber causado exitus por shock hipovolémico.

\section{DISCUSIÓN}

La fístula arterio-ureteral es una causa rara de hematuria macroscópica masiva ${ }^{1,2}$. Algunas fistulas arterio-ureterales se asocian a cirugía vascular previa ${ }^{1-10}$.

Nosotros aportamos un caso en el que la fístula se produce como complicación tras realizar cirugía vascular con colocación de prótesis en segmento iliaco. También observamos otra complicación frecuente en este tipo de cirugía, que es la obstrucción ureteral ${ }^{4,11}$.

Los mecanismos etiopatogénicos parecen ser, por un lado la formación de una fibrosis retrope- 

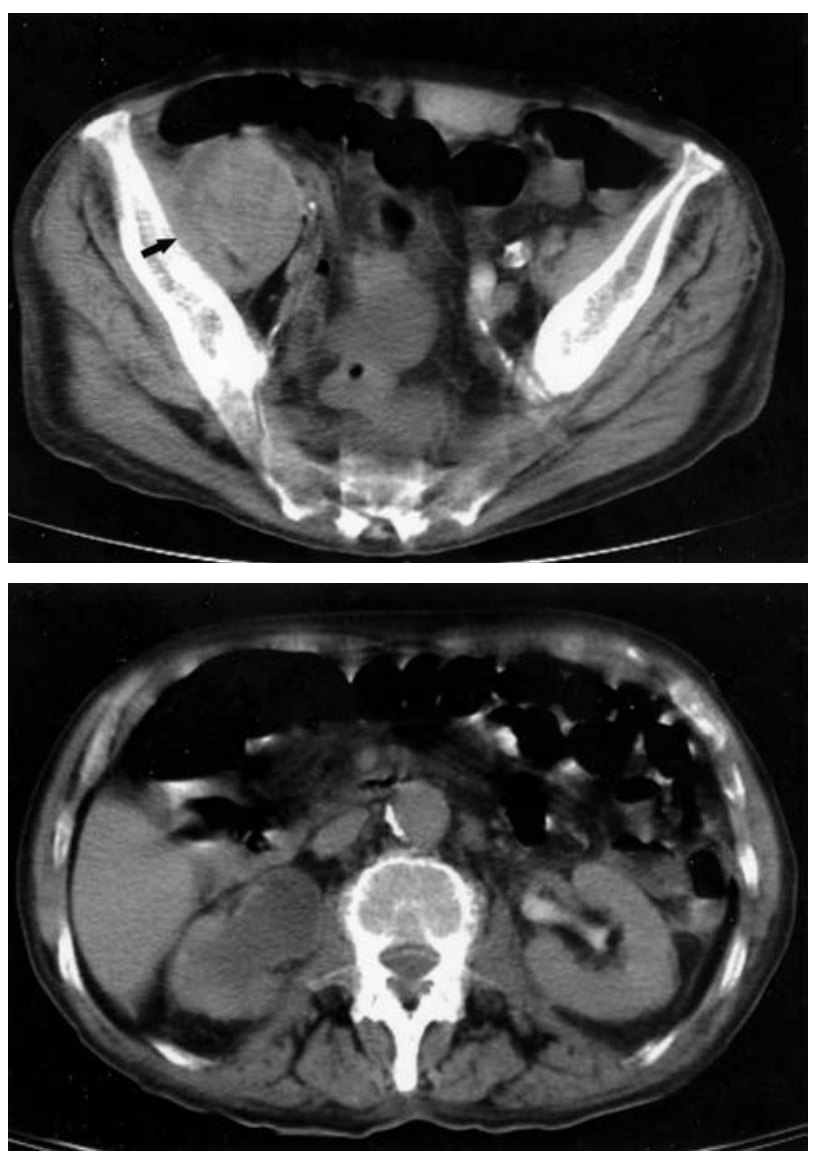

FIGURA 2. TAC: uropatia obstructiva derecha con atrofia renal; pseudoaneurisma de arteria iliaca externa derecha $(6 \mathrm{~cm})$ que implica tercio distal del uréter derecho, con imagen en su interior correspondiente a prótesis vascular.

ritoneal que comprima o englobe al uréter afectado, y por otro, la producción de un decúbito de la prótesis sobre el tejido ureteral que termine produciendo un trayecto fistuloso ${ }^{12}$.

El diagnóstico es difícil y se basa en la sospecha clínica ante un paciente que presente hematuria macroscópica masiva e intermitente, la urografia intravenosa, cistoscopia, ureteropielografia retrógrada, arteriografía y ocasionalmente $\mathrm{TAC}^{1-}$ 10. En nuestro caso el diagnóstico lo confirmó el TAC debido a que la UIV no fue válida por presentar insuficiencia renal y atrofia renal derecha.

El tratamiento es complejo y las opciones van desde actuaciones endourológicas hasta la cirugía abierta (vascular y/o urológica), dependiendo del grado de urgencia y de las posibilidades de reconstrucción ${ }^{3}$. En nuestro caso la opción terapéutica hubiese sido la colocación de endoprótesis vascular que no pudo lograrse por shock hipovolémico y exitus del paciente.

\section{REFERENCIAS}

1. Andreasen JJ, Fahrenkrug L, Madsen PV. Massive hematuria due to iliac artery-ureteral fistula. Eur J Surg 1991;157:223-224.

2. Batter SJ, Mcgovern FJ, Cambria RP. Ureteroarterial fistula: case report and review of the literature. Urology 1996; 48(3):481-489.

3. Simon G, Ballanger P, Midy D, Junes F, Baste JC, Boisieras P. Iliac artery-ureteral fistula after aorto-iliac reconstructive surgery (apropos of 3 cases). Prog Urol 1992 feb;2(1):85-92.

4. Blasco FG, Saladié JM. Ureteral obstruccion and ureteral fistulas after aortofemoral or aortoiliac bypass surgery. $J$ Urol $1991 \mathrm{feb}$; 145:237-242.

5. Akaba N, Ujiie H, Umezawa K, Miura K, Kabayashi S, Sakurai K, Takasaka S. A case of sudden gross hematuria caused by an iliac artery-uretereal fistula. Nippon Geka Gakkai Zasshi 1983 jul;84(7):648-653.

6. Ahlborn TN, Birkhoff JD, Nowygrod R. Common iliac artery-ureteral fistula: case report and literature review. J Vasc Surg 1986 jan;3(1):155-158.

7. Chantada V, Gómez F, Sousa A, González M. Common iliac artery-ureteral fistula: case report and literature review. Arch Esp Urol 1993 nov;46(9):843-846.

8. Krenzien J, Zimmermann HB, Schott H. Iliaco-ureteral fistula and its treatment with stent graft. Chirurg 1998 sept; 69(9):977-980.

9. Van Damme H, Keppenne V, Sakalihasan N, Andrianne R, Limet R. Uretero-arterial fistula: two observations. Acta Chir Belg 1997;97:133-136.

10. Eberle J, Überreiter S, Janetschek G. Uretero-iliac fistula: a rare cause of hematuria. Scand J Urol Nephrol 1992;26:307-309.

11. Ballesteros JJ, Vidal F, Guzmán A, Pares ME. Complicaciones ureterales de la cirugía vascular protésica retroperitoneal. Actas Urol Esp 2001;25(1):55-59.

12. Muruamendiaraz V, García de la Torre A. Las complicaciones ureterales después de la cirugía aorto-ilio-femoral: revisión de 46 casos. Arch Esp Urol 1984;37(1):17-31.

Dr. J.A. Suárez González

Servicio de Urología

Hospital da Costa

C/ Rafael Vior, s/n

27880 Burela (Lugo)

(Trabajo recibido el 9 diciembre de 2003) 